\title{
Performer Type Code
}

National Cancer Institute

\section{Source}

National Cancer Institute. Performer Type Code. NCI Thesaurus. Code C93984.

A coded value specifying the kind of performer. 